\title{
DISTRIBUTION ENERGY STORAGE INVESTMENT PRIORITIZATION WITH A REAL CODED MULTI-OBJECTIVE GENETIC ALGORITHM
}

\author{
Gianni Celli, Fabrizio Pilo, Giuditta Pisano, Gian Giuseppe Soma \\ (celli@diee.unica.it -pilo@diee.unica.it-giuditta.pisano@diee.unica.it-ggsoma@diee.unica.it) \\ University of Cagliari - via Marengo 2, 09123, Cagliari, Italy
}

\begin{abstract}
Energy Storage Systems (ESSs) are progressively becoming an essential requisite for the upcoming Smart Distribution Systems thanks to the flexibility they introduce in the network operation. A rapid improvement in ESS technology efficiency has been seen, but not yet sufficient to drastically reduce the high investments associated. Thus, optimal planning and management of these devices are crucial to identify specific configurations that can justify ESSs installation. This consideration has motivated a strong interest of the researchers in this field that, however, have separately solved the optimal ESS location and the optimal ESS schedule. In the paper, a novel Multi-Objective approach is presented, based on the Non-dominated Sorted Genetic Algorithm - II integrated with a real codification that allows joining in a single optimization all the main features of an optimal ESS implementation project: siting, sizing and scheduling. The methodology has been tested on a realsize rural distribution network.
\end{abstract}

Keywords: Multi-Objective Optimization, Energy Storage System, Distribution Network Planning, Real Coding Genetic Algorithms.

\section{Introduction}

In the last decade, the electric distribution system has started a revolutionary transition towards a "smarter" operation that should reduce costs, enable new services and business opportunities and increase the hosting capacity for renewable energy production and electric vehicles. Flexibility is the key for the success of this transition and it can be provided by several actions, among which the

\footnotetext{
* Corresponding Author: Gianni Celli - phone: +390706755868 - email: celli@diee.unica.it
} 
installation of Energy Storage Systems (ESSs) plays a crucial role [1]. Indeed, the capability of changing behaviour from generator to load depending on variable needs makes the ESS suitable to be employed for a large range of potential applications in the distribution networks, like (but not only) load levelling, renewable energy integration, network congestion relief, voltage control and loss reduction. However, the connection and coordination of an increasing number of ESS also lead to new challenges for the maximum exploitation of their technical and economic potentials, due to the still high costs of installation. Moreover, the solutions to these issues are strictly dependent on the development scenario assumed for the distribution system, e.g. which actors (grid companies, end users, third parties) own and operate ESSs; which business model is supposed; and which services the ESS is enabled to offer. Consequently, several design options can be created and have to be examined, whose number inevitably becomes overwhelming making the decision process hard to be solved. The first step of this decision process is the identification of the optimal ESSs configuration (i.e. location, size and optimal scheduling) with benefits greater than capital and operational expenses.

The energy storage planning in electric distribution network is an optimization problem that has been increasingly attracting the attention of researchers as demonstrated by the high number of papers published, dealing with different combination of multiple synergic applications of the ESS and proposing a variety of models and methodologies [2]-[3]. Due to the "non-deterministic polynomial-time hard" nature of the problem (NP-hard) and the non-linear behaviour of the electric system, the majority of the researchers adopts a meta-heuristic solution method, which does not guarantee finding a global optimal solution, but has proven to be robust (provided solutions are acceptable in practice). Various meta-heuristic methods are used including Genetic Algorithms (GA) [4]-[5], Particle Swarm Optimization [6], Artificial Bee Colony [7] and Differential Evolution [8]. Researchers have also proposed some mathematical programming techniques to find the optimal solution of an approximated model. Particularly, in [9] a mixed integer linear programming (MILP) approach is used to solve an optimal power flow (OPF) that minimizes electricity cost and 
network losses and determines the optimal ESS size and location. In [10] the AC OPF model for radial distribution network is converted to a second order cone programming model (SOCP) and used to optimally locate and dispatch a fixed number of ESS for minimizing investment, maintenance and operational costs. In the vast majority of contributions, benefits and costs of ESS are converted in monetary terms and summed together into a single objective formulation of the problem [4]-[9]. Anyway, monetizing all benefits, even those that are hard to be monetised, for building a weighted combination of economic terms, often implies subjective assumptions and simplifications that can reduce the quality of final results. In this context Multi-Objective (MO) programming is very effective to make the decision process more transparent and impartial, and it can be used for both financial (i.e. company decision making) and socio-economic analysis (e.g., definition of regulation). Currently, few papers present a true MO approach [11], since most of them still uses a weighted sum method [10], [12]. Sometimes, the optimization is focussed on the siting and sizing goals only, because it does not consider objective functions (OFs) dependent on the ESS scheduling [7], [9], [11]. When also this aspect is included, the overall problem is solved by splitting the ESS operation and the ESS planning [6] or by arranging the optimization procedure with nested sub-problems [4]-[5], [8], but always considering a single OF for the optimal ESS scheduling.

In the paper, a full $\mathrm{MO}$ optimization procedure has been developed to identify the Pareto set of design options with fixed network topology for a given MV network. The optimization methodology is based on the Non-dominated Sorting Genetic Algorithm - II (NSGA-II) with a Real Codification of the simultaneously optimized decision variables: the ESS number, the ESS locations (nodes of the MV network), the ESS rating (nominal power and duration), and the daily schedule of the energy stored in each device as well as the cross-section of conductors, and the rated capacity $\mathrm{HV} / \mathrm{MV}$ and MV/LV transformers.

It should be noted that daily schedule of ESS operation is fundamental since the convenience of an ESS can be obtained by only considering the benefits of multiple services at the same time, and not 
with an exclusive operation for a single goal. Thus, it should not be preferential the identification of the daily schedules optimal tailored for specific targets, but the ones of compromise among different targets and overall optimal. The simultaneous optimization of all the ESS features relevant to the set of offered services and the use of multi-objective approach that avoids monetizing all benefits (e.g., externalities and environmental benefits) are advancements with respect to the state of the art. The original codification of the NSGA-II with real quantities, which allows finding the ESS optimal daily scheduling, is another element of novelty compared to existing literature.

\section{Multi-Objective optimization}

The nature of most real-world problems is intrinsically multi-objective. Thus, MO optimization (also called multi-criteria or vector optimization) is become very popular and important for scientists and engineers. Differently from single-objective optimization problems that may have a unique optimal solution, MO problems (as a rule) present a possibly uncountable set of solutions. This set is found by applying the Pareto Optimality Theory. A solution belongs to the Pareto set, or it is said Pareto optimal, if no improvement is possible in one objective without worsening in any other. Thus, the identification of the Pareto is crucial in decision making that looks for fair compromises amongst contrasting needs and stakeholders.

Among the multitude of approaches proposed in Literature, evolutionary algorithms are particularly suitable to solve multi-objective optimization problems. Indeed, they simultaneously deal with a multitude of possible alternatives, (the so-called population) which allows to find an entire set of Pareto optimal solutions in a single run of the algorithm, instead of having to perform a series of separate runs as in the case of the traditional mathematical programming techniques (as the linear combination of weights or the $\varepsilon$-constraint method). Additionally, evolutionary algorithms are less susceptible to the shape or continuity of the Pareto front [13].

Generally speaking, when an evolutionary algorithm is used, two important implementation issues have to be tackle: i) how to assess the quality of each individual (Fitness Function), and ii) how to 
code the solution of the problem for an effective application of the optimization process.

Due to its recognized efficiency and robustness, the NSGA-II technique has been adopted in this paper as optimization engine. It assigns the fitness with a Domination-based approach [14], through the definition of two attributes, the non-domination rank and the crowding distance. The first attribute groups the solutions into different fronts of non-dominance, whereas the second is used to preserve diversity in each Pareto front, by rewarding those solutions located in the less crowded regions of the front.

The solution coding developed for the ESS allocation problem represents one of the main novelties of the paper.

\section{Multi-Objective optimal allocation of Energy Storage Systems}

The traditional binary coding of the Genetic Algorithms (GAs) makes them particularly suitable for solving facility allocation problems. Indeed, in the research field of power distribution system, they have been used extensively for siting and sizing many types of equipment like generators, capacitor banks, measurement and control devices, and in the last decade also ESSs.

For ESSs the optimal allocation problem is even more complex, since the diverse benefits they can provide depend not only on the ESS size and location but also on the daily mode of operation and the share among different functionalities (e.g. energy losses reduction, voltage regulation, peak shaving). Usually, the ESS usage is optimized separately from the main MO optimization for the ESSs siting and sizing. Thus, the scheduling of the energy stored in the ESS is defined as a singleobjective problem, limiting the multi-objective vision of the ESS allocation problem. In order to fill this gap in the Literature, in the paper the ESS daily scheduling has been included in the chromosome used to code the individual.

\subsection{Solution representation}

The chromosome of the generic solution has been organized for including four pieces of information: 
1. the position (MV node) of the ESS in the distribution network;

2. the rated power (expressed in $\mathrm{kW})$;

3. the nominal energy (number of hours at the rated power);

4. the State of Charge (SoC) at the beginning of each hour in the typical day of the year, expressed as percentage of its nominal energy.

Consequently, the chromosome section of a single ESS assumes the representation of Fig. 1. The whole solution vector is obtained by repeating this schema for all the possibly installed devices.

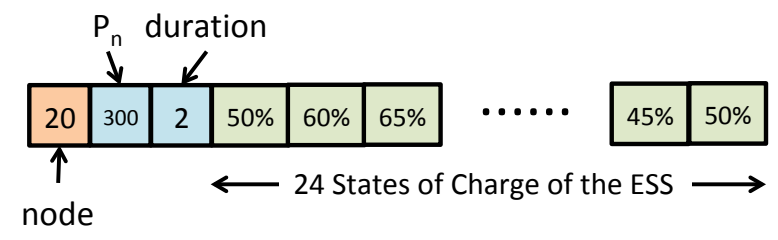

Fig. 1 - Chromosome section for a storage device

It can be observed that the solution space of the multi-objective optimization problem is essentially formed by continuous variables (e.g., SoC of the ESS, nominal power, and duration). Thus, instead of the traditional binary coding, a real-number codification has been adopted, more effective for continuous domains [15]. Indeed, it avoids the concern of an adequate precision of the optimal solutions when the search space is discretized for applying the binary coding. Moreover, Real Coded Genetic Algorithms (RCGAs) have the ability to exploit the gradualness of functions of continuous variables (i.e. small changes in the variables cause small changes in the function).

\subsection{Solution constraints}

During the evolution process, each gene of the chromosome has to remain in its feasible range:

$$
\begin{aligned}
& 1 \leq x_{\text {location }} \leq N_{\text {nodes }} \\
& P_{\text {min }} \leq x_{P n} \leq P_{\max } \\
& d_{\text {min }} \leq x_{d n} \leq d_{\max } \\
& 0 \leq x_{S o C, h} \leq 100 \quad \text { with } \mathrm{h}=1, \ldots, 24
\end{aligned}
$$

where $x_{\text {location }}$ is the position gene, $N_{\text {nodes }}$ the number of nodes in the distribution network, $x_{P n}$ and $x_{d n}$ are the nominal power and the nominal duration genes, $P_{\min }, P_{\max }, d_{\min }$ and $d_{\max }$ are their minimum and maximum limits, fixed by the planner, $x_{S o C, h}$ is the $h^{\text {th }} \mathrm{SoC}$ gene representing the energy stored 
at the beginning of the $h^{\text {th }}$ hour of the day, expressed as percentage of the device nominal energy.

Moreover, two additional constraints have to be satisfied by the SoC genes:

- the difference between the values of two successive genes (consecutive hours) cannot exceed the nominal charging/discharging power (i.e. charge/discharge rate),

- the previous constraint has also to be verified between the first and the last genes, in order to make the scheduling profile repeatable for all the days in a year.

These last two constraints are graphically illustrated in Fig. 2 and mathematically expressed as:

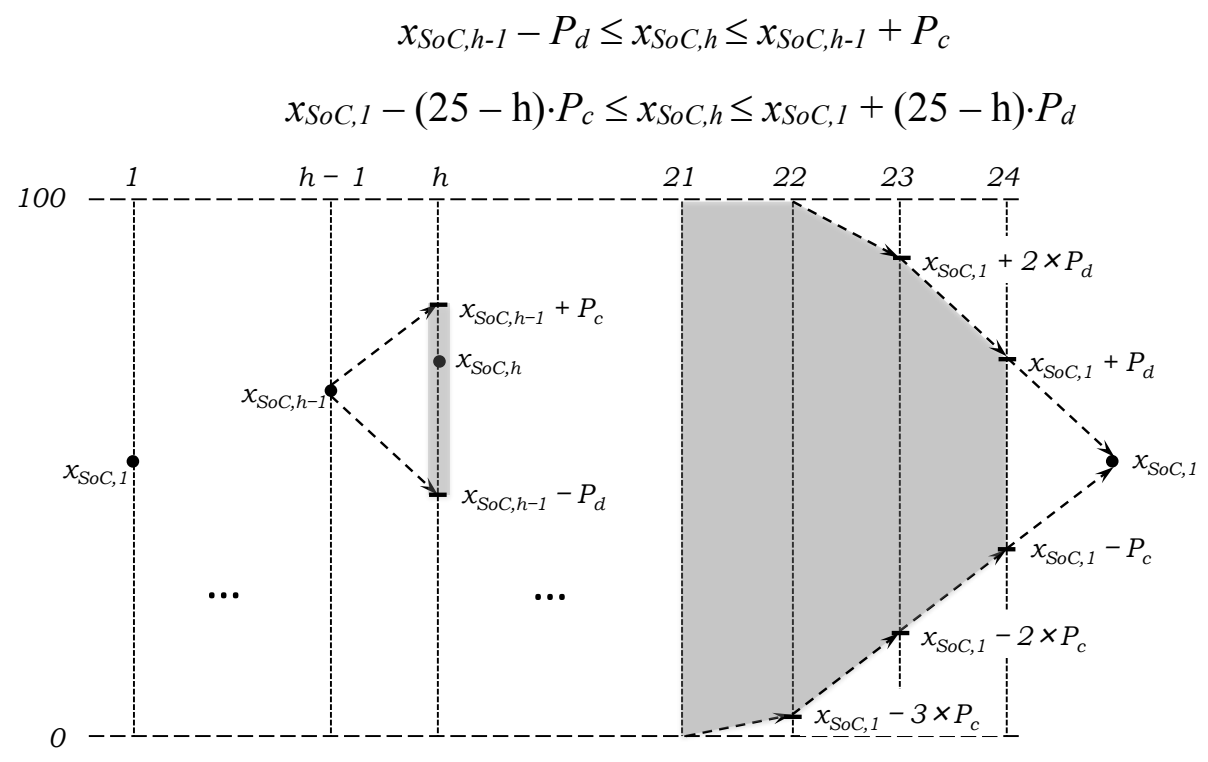

Fig. 2 - Graphic representation of technical and operational constraints for the ESS scheduling

where $P_{c}$ and $P_{d}$ are the maximum charging and discharging power (in the paper assumed equal to nominal power $P_{n}$ ). Since the constraints (4), (5) and (6) have to be satisfied simultaneously, the scheduling profile is bounded by the most stringent for each hour (7).

$$
\max \left\{0, x_{S o C, h-1}-P_{d}, x_{S o C, 1}-(25-\mathrm{h}) \cdot P_{c}\right\} \leq x_{S o C, h} \leq \min \left\{100, x_{S o C, h-1}+P_{c}, x_{S o C, 1}+(25-\mathrm{h}) \cdot P_{d}\right\}
$$

\subsection{Real Coding implementation on the NSGA-II algorithm}

The evolution of the population in the NSGA-II advances similarly to any traditional genetic algorithm. An initial population is created by randomly extracting a real number for each gene of the individuals within the feasible ranges defined by eq. (1), (2), (3), and (7). Then, for each generation the three genetic operators of selection, crossover and mutation are systematically 
applied to produce a new set of offspring solutions [16]. The couples of parents are chosen by using the classical "tournament selection" approach [17]. Instead, the real coding application requires variations of the classical crossover and mutation operators.

\subsubsection{BLX- $\alpha$ crossover operator}

All the mechanisms developed for the binary coding (like two-points or uniform crossover) can be directly used with the real coding. With these mechanisms, the value of a gene in the offspring coincides with the value of this gene in one of the parents. However, the real coding allows introducing new mechanisms (more effective in a continuous domain) based on the numerical combination of the genes values in the parents [18].

These new crossover operators are classified for their capability to implement different degrees of search space exploration and of parents information exploitation [19]. The first property produces additional diversity from the parents that helps exploring the whole search space, whereas the second one uses the existing diversity of the parents for creating better individuals and realizing an improved local search around the previous solutions. These characteristics are represented in Fig. 3 by identifying the regions where offspring gene can be found after the combination of the parents' genes. By indicating with $g_{\min }$ and $g_{\max }$ the smallest and greatest values of the two parents genes and with $[a, b]$ the feasibility range of the gene, three zones are identified: the one between the two parents genes, $\left[g_{\min }, g_{\max }\right]$, is the exploitation zone, because the distance of the offspring gene from both the parents genes is lower than the distance between the latter $(\Delta G)$; the two external intervals are exploration zones because the previous condition is not fulfilled.

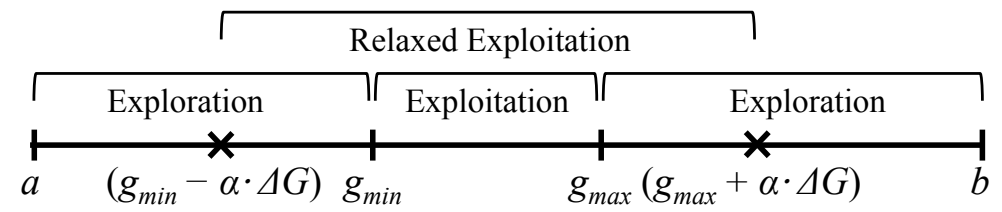

Fig. 3 - Graphical representation of the exploration and exploitation characteristics of a crossover operator for RCGA

In the paper, the Blend Crossover (BLX- $\alpha$ ) operator has been used. It uniformly picks values that lie in the range $\left[g_{\min }-\alpha \cdot \Delta G, g_{\max }+\alpha \cdot \Delta G\right]$. With the parameter $\alpha=0$ the crossover operator realises exploitation only (flat crossover), with $0<\alpha \leq 1$ a relaxed exploitation is obtained, and 
with $\alpha>1$ greater influence is given to the exploration capabilities. The typical value used for this parameter (and adopted in the paper) is $\alpha=0.5$.

For the ESS allocation problem, the extraction interval of the offspring genes has to be included in the constraints (1), (2), (3) and (7) in order to guarantee the individual feasibility (8).

$$
\begin{gathered}
\max \left\{1, g_{\text {min }}-0.5 \cdot \Delta G\right\} \leq x_{\text {location }} \leq \min \left\{N_{\text {nodes }}, g_{\text {max }}+0.5 \cdot \Delta G\right\} \\
\max \left\{P_{\min }, g_{\min }-0.5 \cdot \Delta G\right\} \leq x_{P n} \leq \min \left\{P_{\max }, g_{\max }+0.5 \cdot \Delta G\right\} \\
\max \left\{d_{\min }, g_{\min }-0.5 \cdot \Delta G\right\} \leq x_{d n} \leq \min \left\{d_{\max }, g_{\max }+0.5 \cdot \Delta G\right\} \\
\max \left\{0, x_{S o C, h-1}-P_{d}, x_{S o C, I}-(25-\mathrm{h}) \cdot P_{c}, g_{\text {min }}-0.5 \cdot \Delta G\right\} \leq x_{S o C, h} \leq \min \left\{100, x_{S o C, h-1}+P_{c}, x_{S o C, I}+(25-\mathrm{h}) \cdot P_{d}, g_{\text {max }}+0.5 \cdot \Delta G\right\}
\end{gathered}
$$

\subsubsection{Non-uniform mutation}

Several mutation operators for RCGAs, which differ for the probability distribution used to perturb the value of the selected gene (random, Gaussian, polynomial, etc.) and for the mutation scheme adopted for selecting the gene to mutate, have been proposed in the Literature. In the paper, the non-uniform mutation and the mutation clock scheme are implemented, because they result among the most performing approaches for RCGAs [18], [20].

The non-uniform mutation uses a polynomial probability distribution to change the current variable value (parent) to a neighbouring value (offspring). By indicating with $c_{g} \in\left[a_{g}, b_{g}\right]$ the value of the $g^{\text {th }}$ gene to mutate, the new value $\left(c^{\prime} g\right)$ is obtained from (9).

$$
c_{g}^{\prime}=\left\{\begin{array}{ll}
c_{g}+\Delta\left(t, b_{g}-c_{g}\right) & \text { if } \tau=0 \\
c_{g}-\Delta\left(t, c_{g}-a_{g}\right) & \text { if } \tau=1
\end{array} \quad \text { with } \Delta(t, y)=y \cdot\left[1-r^{\wedge}(1-t / M A X G E N)^{\wedge} \beta\right]\right.
$$

$M A X G E N$ is the total number of generations, $t$ is the generic generation, $\tau$ is a random binary number, $r$ is a random real number in the interval $[0,1]$, and $\beta$ is a parameter used to fix the dependency on the number of generations. In this way, $c_{g}^{\prime}$ is randomly extracted within a progressively reduced interval, causing the mutation operator to make a uniform search in the initial stage when $t$ is small, and a calibrated search at later stages, favouring local tuning (Fig. 4).
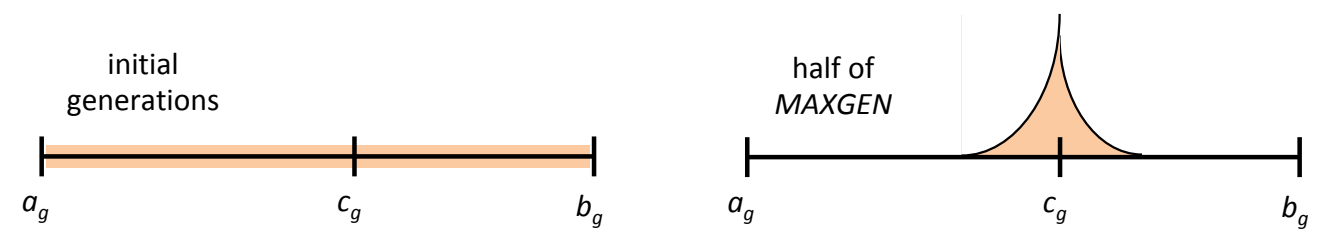

Fig. 4 - Probability density function of creating a mutated offspring gene from a parent gene $c_{g}$ 
The parameters used in the paper for the non-uniform mutation operator are summarized in Table 1.

Table 1 - Parameters used for the nun-uniform mutation operator

\begin{tabular}{cccc}
\hline Gene & $\boldsymbol{a}_{\boldsymbol{g}}$ & $\boldsymbol{b}_{\boldsymbol{g}}$ & $\boldsymbol{\beta}$ \\
\hline$x_{\text {location }}$ & 1 & $N_{\text {nodes }}$ & 2 \\
$x_{P n}$ & $P_{\text {min }}$ & $P_{\max }$ & 5 \\
$x_{d n}$ & $d_{\min }$ & $d_{\max }$ & 5 \\
$x_{S o C, h}$ & $\max \left\{0, x_{S o C, h-1}-P_{d}, x_{S o C, h+1}-P_{c}\right\}$ & $\min \left\{100, x_{S o C, h-1}+P_{c}, x_{S o C, h+1}+P_{d}\right\}$ & 5 \\
\hline
\end{tabular}

The smaller value for the exponent $\beta$ in case of the location gene is justified by the low importance of the local tuning, because the numeration of the distribution network nodes does not guarantee that the local search would be limited to the adjacent nodes.

The usual mutation scheme adopted for the gene selection is based on a mutation probability $p_{m}$ equal to the inverse of the number $n$ of genes in the chromosome and requires one random extraction for each gene ( $n$ extractions for each individual). Differently, mutation clock scheme reduces the computational burden with the same mutation probability, because on average it needs one extraction per individual. Indeed, once a gene is mutated, the next selection is chosen by using an exponential probability distribution: the following occurrence of mutation $(l)$ is obtained as $l=-\left(p_{m}\right)^{-1} \cdot \log (1-u)$, where $u$ is a random number in the unity interval $[0,1]$. Thus, if the $g^{\text {th }}$ gene of the $s^{\text {th }}$ individual is currently mutated, the next gene to be mutated is the $[(g+l) \bmod n]^{\text {th }}$ of the $\{s+\operatorname{int}[(g+l) / n]\}^{\text {th }}$ individual. At each generation, the first mutation is found by using $s=g=1$.

\section{Objective Functions}

Several benefits can be associated to the installation of ESSs in the electric distribution networks. Apart from an ESS operation devoted to private incomes (arbitrage), benefits can be distinguished between those oriented towards the provision of ancillary services to the transmission system (primary and secondary frequency regulation, reactive power compensation at the transmission system interface, black start), and those that directly interest the distribution system operator (DSO). In the paper, only the latter have been considered. Some of these benefits have an impact on the network operation and the quality of service provided (e.g, Joule energy losses reduction, continuity 
of supply enhancement, voltage regulation improvement, voltage dips mitigation); some others influence the network planning (deferral of network investments, hosting capacity increment). To be correctly assessed all benefits require specific network calculations. A brief description of this calculation is firstly provided, followed by the formal definitions of the used objective functions.

\subsection{Probabilistic network calculation}

The development of the future distribution networks requires the implementation of a risk-based approach in replacement of the traditional "fit and forget" methodology hitherto followed from most of the DSOs [21]. Following this idea, the authors have developed in the past decades a planning tool for the optimal integration of Distributed Energy Resources (DER) in the distribution networks. This software is based on a Probabilistic Load Flow (PLF) solved for the 24 hours of one or more typical days both in steady state and emergency network configurations (the latter obtained removing one network element at a time in an $\mathrm{N}-1$ analysis). The daily load/generation profiles are characterised in each hour with a mean value and a standard deviation (assumption of Gaussian distribution). The result of the calculation is the probability density function (pdf) of the nodal voltages and of the branch currents, used to assess the risk to have nodal voltages out of the regulation band and current beyond the thermal limit. Thus, network upgrade investments are not decided by finding a priori the worst operating conditions (maximum generation - minimum load, maximum load - no generation), but by comparing the risk of the technical constraints violation with the maximum level of acceptable risk [22].

Fig. 5 shows the flow chart of the network calculation procedure with the integration of ESSs. 


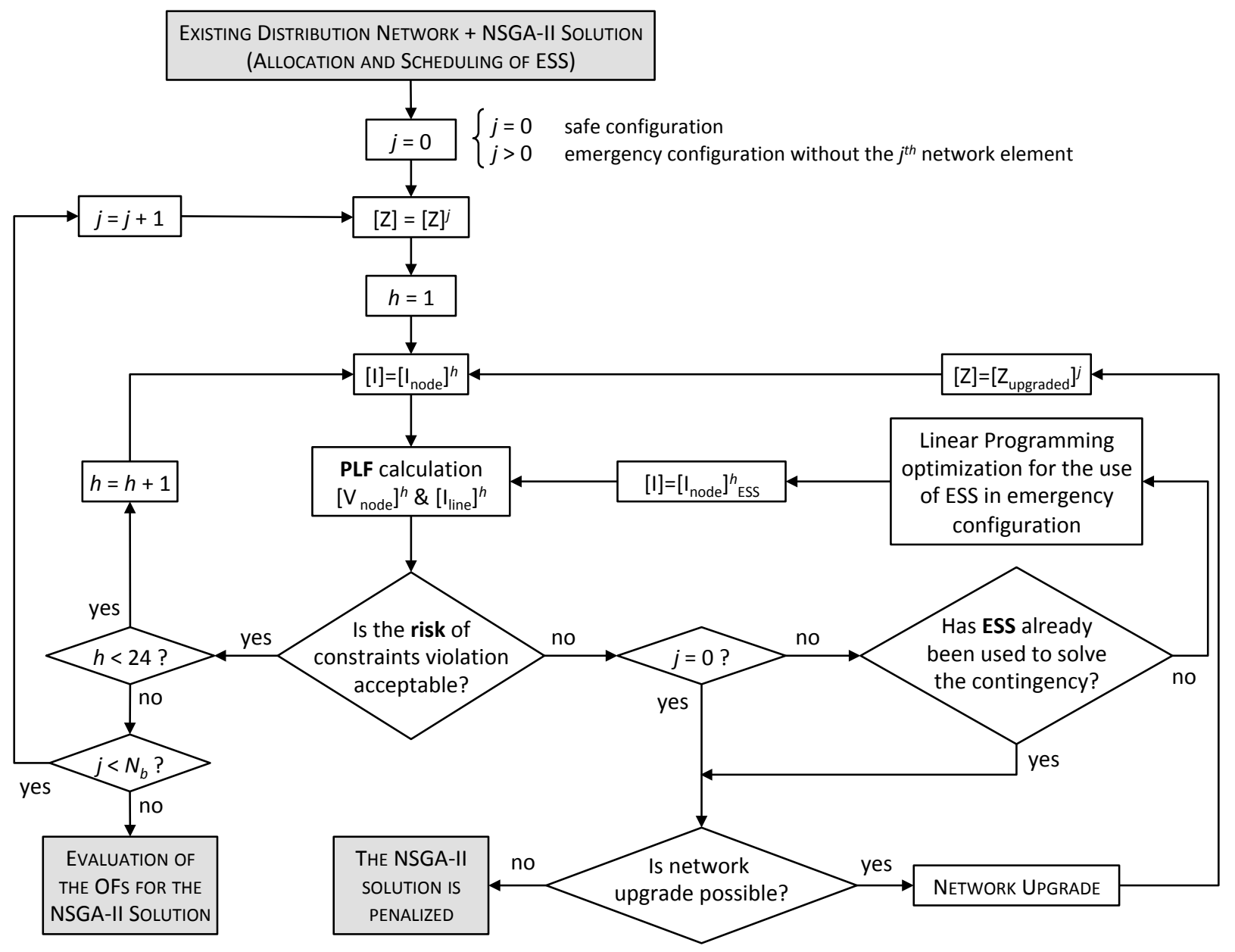

Fig. 5 - Flow chart of the network calculation procedure in presence of ESS

For each hour, the PLF is executed and the risk of technical constraints violation is calculated.

Whether the risk is acceptable the procedure advances to the next hour otherwise the network must be revamped. When all the technical constraints are satisfied with an acceptable risk for all hours of a typical day in all the possible network configurations, the value of OFs are calculated. Otherwise, the design alternative is penalised with the lowest rank in the fitness assignment within the NSGAII algorithm. Regarding the ESS probabilistic representation, the mean value of the active power is directly derived from scheduling. Instead, the standard deviation is estimated in each hour on the basis of the difference between the nominal power of the storage device and the actual power exchanged with the network in that hour. The reactive power has been represented with a zero mean value and a standard deviation equal to one-third of the nominal power (with the assumption of the inverter oversizing in order to decouple active and reactive power flows). In this case the standard deviations do not model uncertainties, but the effect of the voltage regulation service provided by 
the ESS. Indeed, the flexibility introduced by the storage can be used to compensate the voltage variations that can appear in the feeder due to the unpredictable behaviour of loads and generators. Thus, the ESSs have been represented in the PLF equations with a negative linear dependence on respect to the other customers. By so doing, the standard deviations introduced by the ESSs tends to reduce the standard deviations of the nodal voltages in the feeder where the storage is installed, improving the regulation, reducing the risk of constraint violation and, indirectly, increasing the hosting capacity of the distribution system [23].

Only for emergency configuration, when the risk of technical constraints violation is unacceptable, the ESS is used to provide support to the network, deviating from its scheduled energy profile. Indeed, it is reasonable that the DSO uses storage devices to improve the operation when the network is reconfigured due to an outage. Obviously, the effectiveness of this service is contingent on the amount of energy stored at the time of the reconfiguration. For instance, the ESS should store and save enough energy to locally supply the load during the repair time of a faulted network element to avoid the overload of reconfigured feeders. This service is particular important for the deferral of network investments, because most of them are motivated for constraints violations caused by post fault actions. A simple linear programming algorithm has been implemented to find the proper management of the storage device in these conditions [22].

\section{2 $\mathrm{OF}_{1}$ : deferral of network investments}

The ESS may contribute to limit the occurrence of contingencies in the distribution network, both in normal and emergency conditions, by compensating the excessive production from renewable generation or the high demand when the generation is insufficient.

The need for network upgrades is checked with the network calculations for the first and the last years of each sub-period into which the whole planning period has been divided (this division is usually done when new loads or generators are connected). Indeed, with the assumption of constant load growth rate and fixed generation in a sub-period, the first year has the highest probability of 
contingencies for excessive generation, while the last year has the highest probability of contingencies for excessive demand.

Network investment, $C_{U}$, is calculated by summing the building and the maintenance costs of the new elements $\left(B_{0 j}\right.$ and $\left.M_{0 j}\right)$ and by subtracting the eventual residual values $\left(R_{0 j}\right)$ in case the lifespan of the $j^{\text {th }}$ network element is greater than the planning period duration. All the costs are actualized at the beginning of the planning period (Net Present Values) through a prefixed discount rate.

$$
C_{U}=\sum_{j=1 \ldots N b}\left(B_{0 j}+M_{0 j}-R_{0 j}\right)
$$

$N_{b}$ is the number of branches in the distribution network.

\section{3 $\mathrm{OF}_{2}$ : Reduction of Joule energy losses}

The customer demand profile has been modelled as a piecewise linear curve, with the load growth rate that may be different in each sub-period. Due to this statement, it is acceptable for planning studies to assume that the branch current grows linearly also. The Joule energy losses $\left(E_{L}\right)$ in the network are calculated with (11).

$$
\begin{gathered}
E_{L}=\sum_{j=1 \ldots N b} \sum_{k=1 \ldots N s p}\left(E_{L j k}\right)=(8760 / 1000) \cdot \sum_{j=1 \ldots N b} 3 \cdot r_{j} \cdot L_{j} \cdot \sum_{k=1 \ldots N s p}\left(\int_{0} N k I_{j k}{ }^{2}\right)=26.28 \cdot \sum_{j=1 \ldots N b} r_{j} \cdot L_{j} \cdot \\
{\left[\sum_{k=1 \ldots N s p} N_{k} \cdot\left(I_{j j k^{2}}+I_{j j k}{ }^{2}+I_{j j k} \cdot I_{0 j k}\right)\right]}
\end{gathered}
$$

$I_{0 j k}$ and $I_{f j k}$ are respectively the currents of the $j^{\text {th }}$ branch at the beginning and at the end of the $k^{\text {th }}$ sub-period, $N_{k}$ is the sub-period duration in years, $N_{s p}$ is the number of sub-periods, $r_{j}$ and $L_{j}$ are respectively the conductor resistance per kilometre and the length $(\mathrm{km})$ of the $j^{\text {th }}$ branch.

\section{4 $\mathrm{OF}_{3}$ : Improvement of voltage regulation}

The goal is not the reduction of the voltage constrain violations (already included implicitly in the investment deferral for network upgrade) but the improvement of the voltage profile quality achievable with the ESS. The proposed metric is a measure of the maximum nodal voltage variability in the whole network in the last year of the planning period, estimated with the nodal voltage probability distribution obtained with the PLF. This index has been defined with (12).

$$
V_{\text {reg }}=\sum_{i=1 \ldots \text { Nnodes }} \sum_{h=1 \ldots 24}\left(V_{i, h}{ }^{\max }-V_{i, h}{ }^{\min }\right)
$$


In (12) the maximum and minimum voltages in each node in every hour are obtained by adding or subtracting to the mean value three times the standard deviation respectively.

\section{5 $\mathrm{OF}_{4}$ : Improvement of continuity of supply}

ESS may be used to improve the distribution network reliability with the intentional islanding service that allows avoiding long interruptions to customers supplied by faulted laterals (usually, the trunk nodes can be energised entirely with tie lines).

The metric adopted is the System Average Interruption Duration Index (SAIDI) defined as in (13).

$$
\text { SAIDI }=\left(\sum_{i=1 \ldots \text { Nnodes }}\left(U_{i} \cdot N C_{i}\right) / \sum_{i=1 \ldots \text { Nnodes }} N C_{i}\right)
$$

$U_{i}$ and $N C_{i}$ are the annual outage duration and the number of customers of the $i^{t h}$ node.

During the N-1 calculations isolated islands are checked. If the ESSs power rate in the islanded portion is greater than the maximum power requested from the islanded area, and the SoC of ESSs in the examined hour is adequate to provide the service, the intentional islanding is admitted. This is repeated in order to calculate the duration of islanded condition and the relative effect on SAIDI.

\subsection{OF 5 : Reduction of voltage dips}

It has been assumed that the ESS can be temporarily operated as a Dynamic Voltage Restorer to hold up the voltages in the neighbouring nodes of its connection point by injecting the needed power. The metric used to measure this objective is the sum of the annual cumulative frequency of voltage dips in every network nodes, estimated by using the fault location method that exploits the knowledge of fault rates and network impedance matrix for assessing the nodal voltages during any kind of fault (i.e. symmetric and asymmetric, transient and permanent) [24]. By comparing these voltages with the threshold on voltage dip depth (fixed in the paper to $50 \%$ of the nominal voltage), the number of dangerous events in every network node can be enumerated. $\mathrm{OF}_{5}$ typically represents a power service due to the short duration of the voltage dip and it is activated only in case of a fault (it does not affect, and it is not affected by, the daily schedule of the stored energy).

\subsection{OF 6 : Cost of Energy Storage System}


The ESS costs have been divided into the physical installation costs (CAPEX) and annual charge/discharge losses due to the round-trip efficiency (OPEX). An ESS is formed essentially by the storage system and the power conversion system. Thus, the CAPEX of an ESS is usually given by two terms, one reliant on the power rating and the other dependent on the nominal capacity.

$$
C_{C A P E X}{ }^{E S S}=\left(k_{P C S} \cdot c_{p} \cdot P_{n}\right)+\left(c_{e} \cdot P_{n} \cdot d_{n}\right)
$$

where $c_{p}$ and $c_{e}$ are the specific costs of the ESS adopted technology, and $k_{P C S}$ is the oversizing factor of the power conversion system needed to decouple the active and reactive power operation. The maintenance cost is usually small, and it has been disregarded in the paper. As for the network investments, the lifespan of the ESS has been compared with the planning period duration in order to assess the residual value and/or the need to reinvest on a new device.

The ESS energy losses, OPEX, have been summed to $\mathrm{OF}_{2}$.

\section{Case Study and Discussion}

The case study network (Fig. 6) has been derived from the Italian reference rural distribution network, identified by the ATLANTIDE project [25]. This network consists in $102 \mathrm{MV}$ nodes (16 MV customers and $86 \mathrm{MV} / \mathrm{LV}$ substations) supplied by one $25 \mathrm{MVA} 132 / 20 \mathrm{kV}$ transformer and disposed on 7 feeders of different lengths (mostly small cross section overhead conductors for a total extension of about $160 \mathrm{~km}$ ), the longest being around $23 \mathrm{~km}$. As usual in many countries, the MV network is based on a balanced three-wires distribution and it is organized in trunk and lateral nodes. The first ones can be always re-supplied in case of a fault in the upward portion of the feeder through tie lines from neighbouring primary substations or from different feeders of the same primary substation. The laterals have normally a pure radial network topology, even if additional tie lines may exist. The generation scenario is characterized by an intensive presence of photovoltaic (PV) plants, which achieve a total amount of 34 MW. The load - a mix of LV agricultural, residential and small MV customers (as dairy farms) - is about $18.8 \mathrm{MW}$ at the peak, with a power factor 0.9 lagging, and it grows in the 10 years of the considered planning period with a linear yearly rate, respectively for the three cited load categories, of $1.6 \%, 0.4 \%$ and $0.6 \%$. 


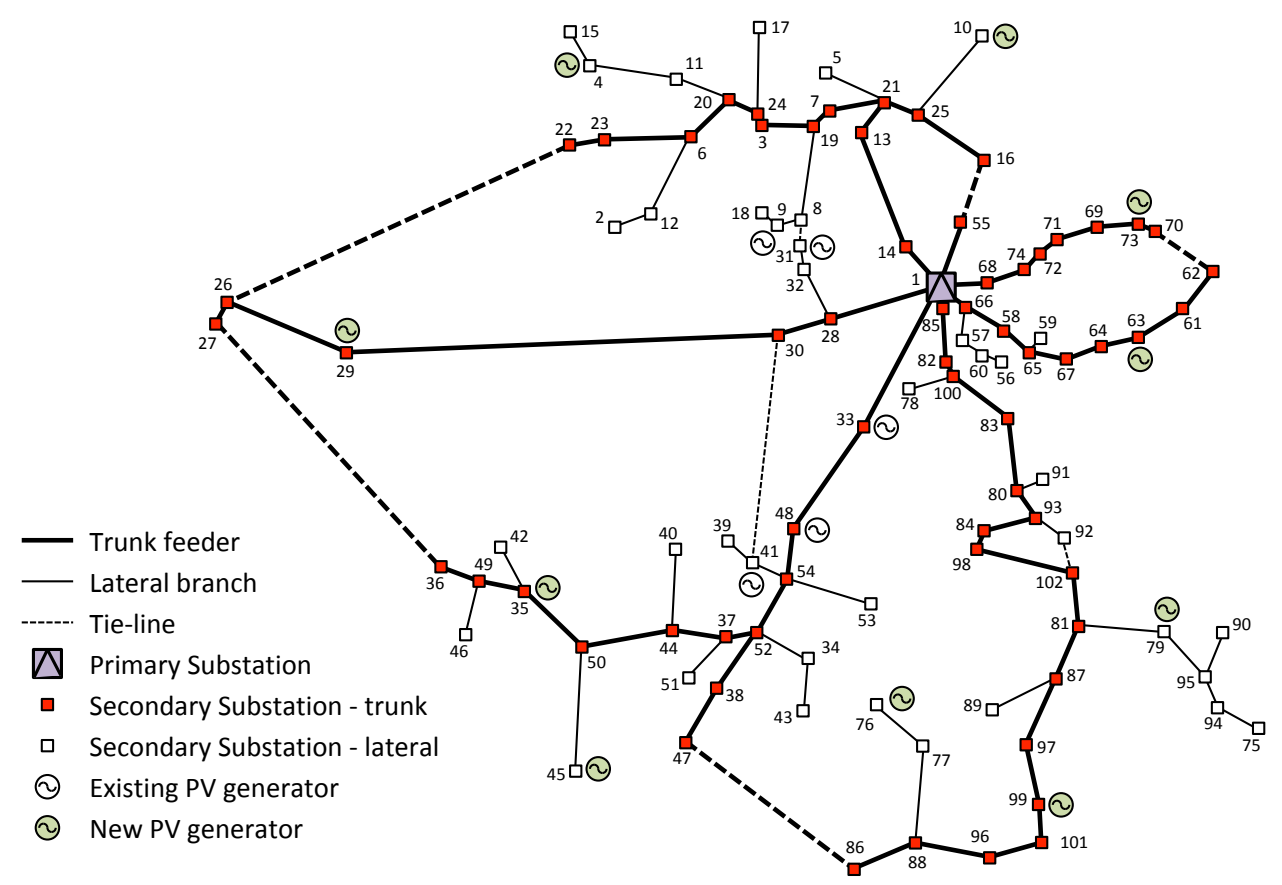

Fig. 6-MV case study network

Generally, the low density of the electric demand in MV rural distribution does not cause overload issues. On the contrary, the long distances covered can determine frequent voltage variation issues during the evening peak demand and the daytime hours of peak PV production, particularly when the network has been reconfigured due to an element outage. Indeed, in emergency configurations, some nodes can be located more than $40 \mathrm{~km}$ far from the primary substation. This technical issue is traditionally solved with network investments. Within this planning framework, the performances of the case study in the whole planning period are summarized in Table 2.

The Smart Grid paradigm proposes to fix operational issues also by resorting to an appropriate management of DER available in the distribution system (non-network solutions). For the sake of simplicity, only the ESSs management has been considered, with the other resources (generators and loads) not involved in the control. With these assumptions, the described methodology has been applied to the case study in order to find optimal projects of storage installation. The ESS technology considered is Li-Ion battery with a lifespan of 10 years, $c_{p}=200 € / \mathrm{kW}$ and $c_{e}=400$ $€ / \mathrm{kWh}$. The nominal power has been chosen in the range $100 \mathrm{~kW} \div 3 \mathrm{MW}$; nominal duration between 1 and 8 hours. All nodes are eligible for ESSs. 
Table 2 - Objective Functions evaluation for the case study in the planning period without ESS

\begin{tabular}{cl}
\hline Objective Function & \multicolumn{1}{c}{ Performance } \\
\hline $\mathrm{OF}_{1}$ & $6428.9[\mathrm{k} €]$ \\
$\mathrm{OF}_{2}$ & $6862.9[\mathrm{MWh}]$ \\
$\mathrm{OF}_{3}$ & $18.7[\mathrm{p} . \mathrm{u}]$. \\
$\mathrm{OF}_{4}$ & $0.923[$ hours/year·customer] \\
$\mathrm{OF}_{5}$ & $1960[$ voltage dips/year] \\
\hline
\end{tabular}

Given the huge number of combinations available for the definition of a generic solution and taking into account the general rule for the GAs of increasing the population size and the maximum number of generations proportionally to the dimension of the optimization problem, a population of 1,000 individuals and a maximum number of 1,000 generations have been chosen. The enormous amount of calculations needed for the correct assessment of the ESSs impact on the distribution system (execution of a probabilistic load flow for each hour of the typical day both in ordinary network conditions and for all the $\mathrm{N}-1$ emergency configurations) has determined a significant computational time of about ten days in a workstation Intel ${ }^{\circledR}$ Xeon ${ }^{\circledR}$ CPU E5 at $3.60 \mathrm{GHz}(\mathrm{RAM}$ 64 GB). This aspect represents the weakest point of the methodology, even if for network planning purposes speediness is not a primary goal. In any case, further research is currently in progress to speed up the procedure, by following two directions: the software parallelization, to exploit the actual CPU and GPU multi-core architecture, and the exploration of the new decomposition-based MO evolutionary optimization techniques [26] that seem more effective for solving many objective optimization problems, requiring less generations to achieve good quality solutions.

Once completed the optimization, all the individuals belonging to the Pareto set differ for number of installed ESSs (on average 3 devices per solution), allocation points, rates and daily schedules of stored energy. From the analysis of the allocation points, every node may be used as possible candidate, even if it is evident the trend of siting the storage devices near the generators or in the main laterals (Fig. 7), in order to limit extreme voltage variations and avoid network upgrades. 


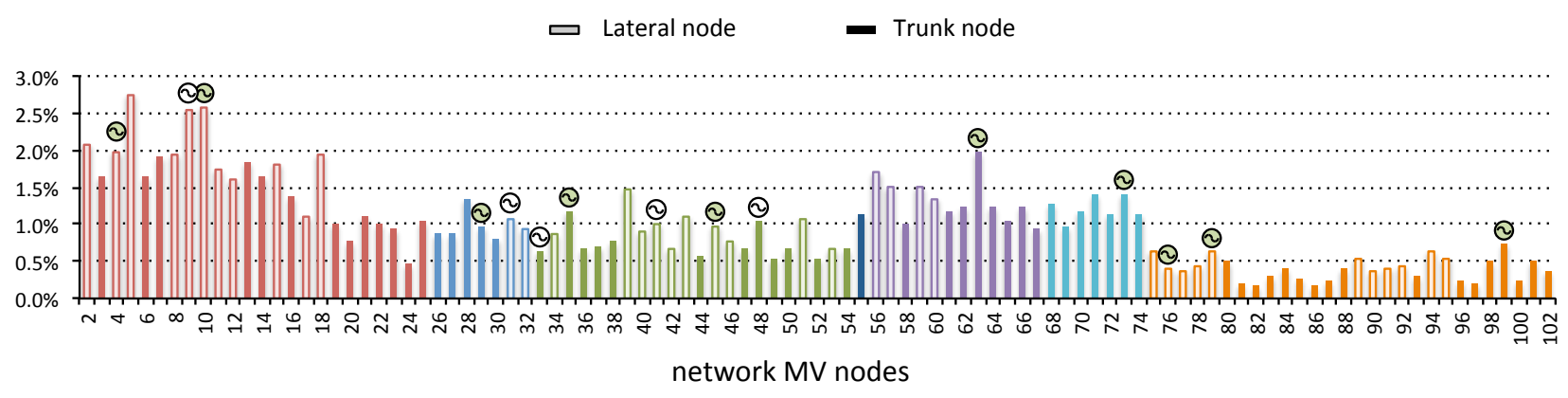

Fig. 7 - Occurrence probability of ESS siting among MV distribution network nodes (feeders marked with different colours)

The most frequent size of the ESS is in the range $0.5 \div 1.5 \mathrm{MW}$ and $5 \div 8$ hours (Fig. 8). This result confirms that, for a rural distribution system, usually high peak power services are not needed. Furthermore, a bigger capacity of storing energy is preferable to successfully provide network support during the operation of emergency configurations.

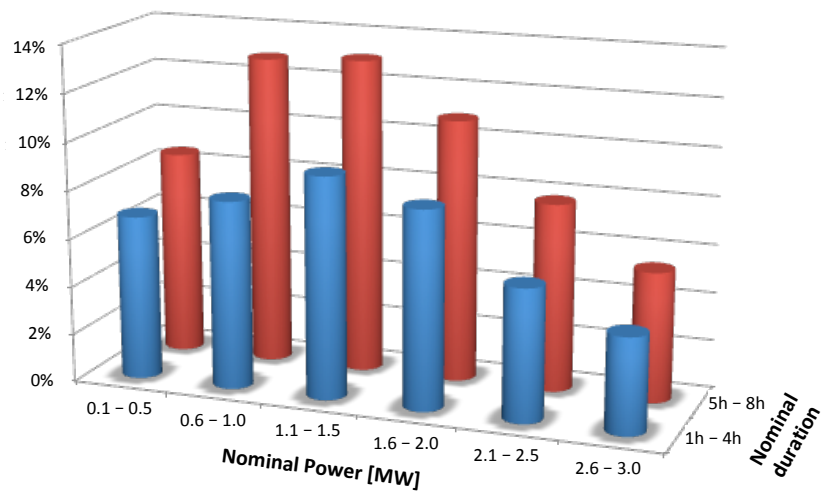

Fig. 8 - Occurrence probability of ESS rating

More than the previous analysed features, the daily schedule of stored energy has been the main reason of diversification among the Pareto solutions, because minor adjustments in the exchanged power lead to slight variations in continuous OFs (like the Joule losses reduction). Consequently, each schedule differs from any other. It ranges from profiles more tailored to specific objectives to those adapted for finding a good compromise among different goals. Moreover, the same OF can be improved with a different operation of the storage devices, depending on their size, the location in the network and, accordingly, on the specific features of local demand and generation. For these reasons, the selection of optimal daily schedules is not unique, even if some general considerations can be done. Fig. 9 shows the different sorting of the Pareto set for each OF. 


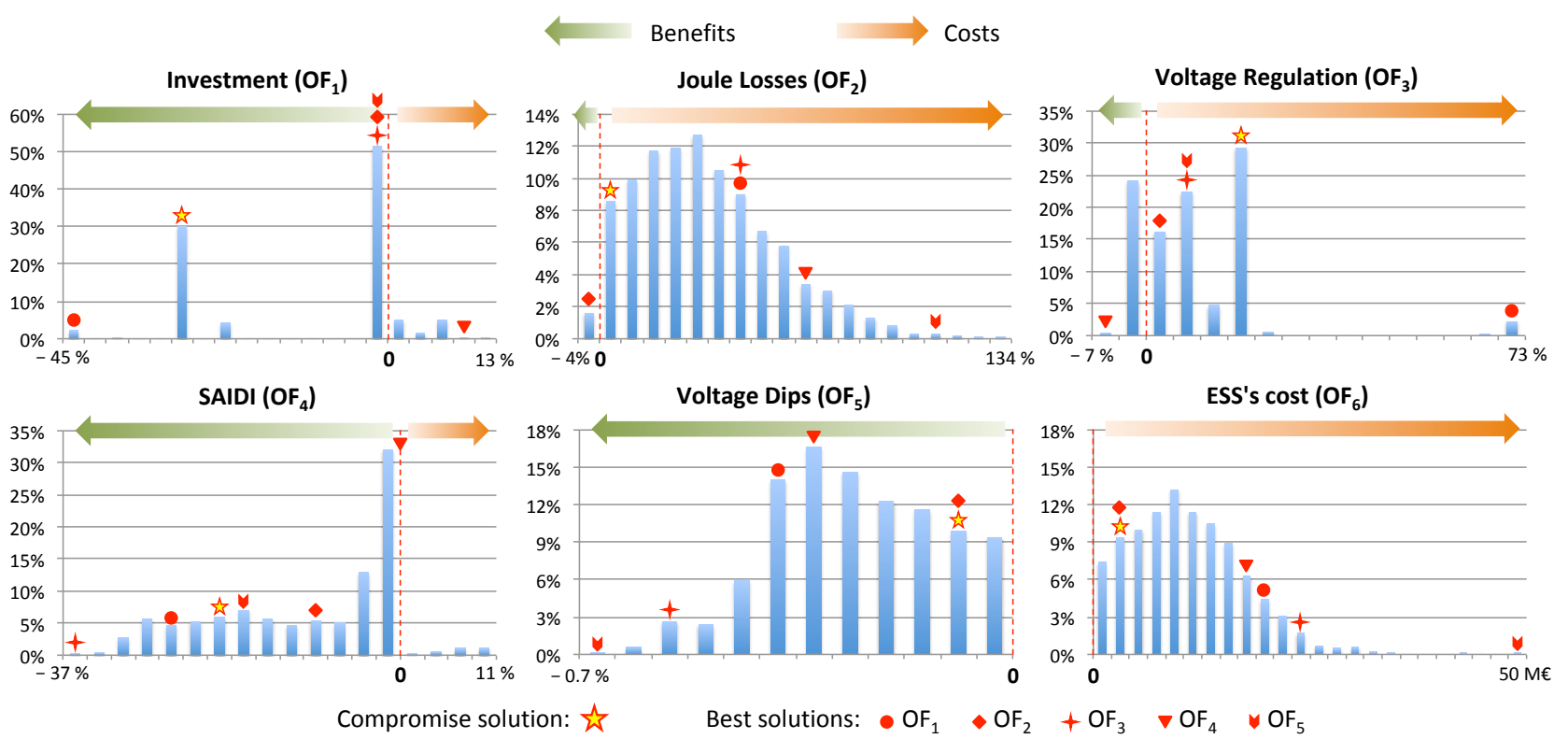

Fig. 9 -Distributions of Pareto solutions with respect to each Objective Function (the zero points in the horizontal axes represent the performance of the distribution system without ESSs installed)

The histograms have been built considering the performance variation with respect to the network planned without ESS. In this way, it is easy to distinguish the cases of performance improvement (i.e. benefits, like for instance the reduction of the percentage of network investments) from those of worsening (costs). From a first analysis, it is evident the strong discontinuity of the $\mathrm{OF}_{1}$ (investment), which depends on the few discrete values of the conductor cross-sections usually available for the lines upgrade. Thus, many solutions (different in the allocation nodes, storage rates and daily schedule) produce the same performance. Almost $35 \%$ of the optimal solutions allows a significant reduction of the network investments that would be needed if no ESS is installed (30 $45 \%$, about $2 \div 3 \mathrm{M} €$ ), while more than $50 \%$ gives slight savings or has no effects. The main savings are not achieved through the peak shaving services during normal operating conditions, but thanks to the ESS support provided during emergency configurations. For this reason, several daily energy profiles are characterized by small changing patterns (almost flat for many hours), confined within the central-upper or the central-lower regions of the ESS daily capability of storing energy, depending respectively on the local dominance of demand or generation (Fig. 10-a). Indeed, these operations allow providing, in any moment of the day after a fault, the required energy reserve to sustain the voltage of the reconfigured feeder (Fig. 10-b). 

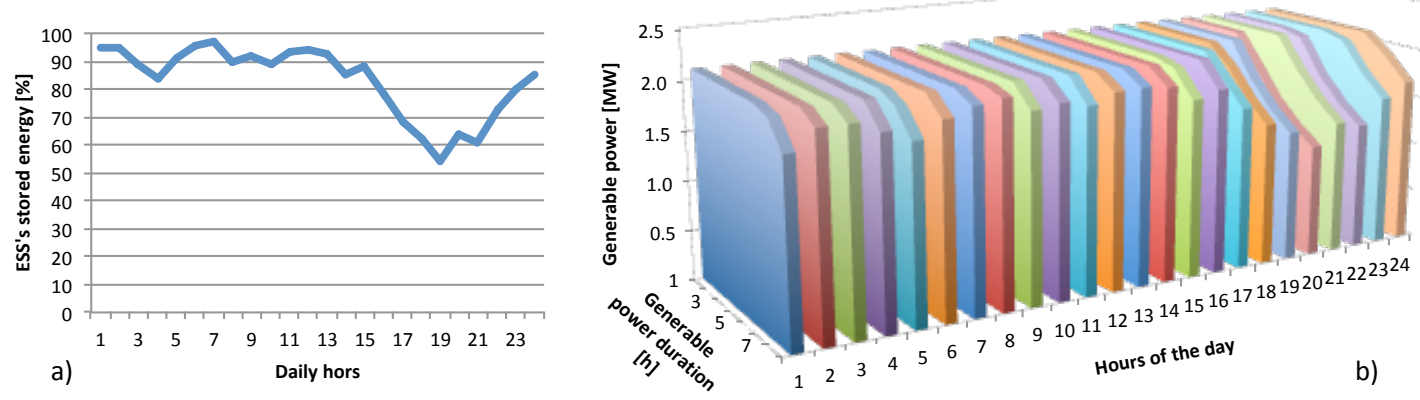

Fig. 10 - Example extracted from one of the best ESS allocations for network investment reduction (storage device of $2.1 \mathrm{MW}-8 \mathrm{~h}$, installed on node 62): a) stored energy profile, b) generable power duration for each hour of the day

The same feature is useful also to reduce the SAIDI index $\left(\mathrm{OF}_{4}\right)$ thanks to the intentional islanding. However, this similarity does not guarantee a full convergence of the two goals, because the best locations to maximize the network investment reduction are at the trunks ends, whereas the best solutions for the reliability improvement is obtained with ESSs installed on laterals.

At first sight, network efficiency $\left(\mathrm{OF}_{2}\right)$ and voltage regulation $\left(\mathrm{OF}_{3}\right)$ do not seem to benefit from the presence of ESSs. Indeed, the majority of the Pareto solutions (particularly for $\mathrm{OF}_{2}$ ) causes a worsening of the network performances. This statement is not completely true. Clearly, at equal network topology and conductors size, an ESS suitably located and operated is able to decrease energy losses or limit nodal voltage variations. However, these results contrast the reduction of network investments, because they strictly depend on the network impedance. The lower is the conductor cross-section the higher the losses and the voltage deviations. Moreover, it must be observed that the reactive power support provided by the ESS in the voltage regulation is fundamental for the technical feasibility of network investments reductions. Without ESS it would not be possible to reduce investments and keep voltage quality within an acceptable band. For instance, with the compromise choice proposed in the paper, the reduction of CAPEX is dramatic, and the worsening of voltage quality is relatively small thanks to the ESS installed. It confirms that ESS enables the maximum exploitation of the existing distribution assets (i.e. the increasing of hosting capacity) by minimizing the negative impacts in terms of efficiency and voltage quality. The effect of ESSs on voltage dips cutback appears marginal, if compared with the other goals. Indeed, ideally, it requires the installation of a storage device for every node of the distribution 
system or the installation of several high power ESSs in order to protect as many nodes as possible. For this reason, the best solution of $\mathrm{OF}_{5}$ is also the one with the highest ESS cost.

Finding a compromise solution among these contrasting goals is a hard task that depends on the technological advance of the batteries and their cost evolution, the planner needs, and the regulatory framework. The most neutral choice falls in the knee of the Pareto frontier, by assuming an equal weight for each objective function (yellow star in Fig. 9). It is characterized by a single $1 \mathrm{MW}-7$ hours ESS connected to the lateral node 11. This ESS allows the connection of the new generator of node 4 , preserving the existing asset (31\% of saving on network CAPEX) and, simultaneously, enabling the intentional islanding on the lateral (19\% of SAIDI reduction). The storage is normally operated by absorbing energy at daytime (with high generation) and releasing energy during the evening (peak demand). By so doing, the ESS limits the increment of Joule losses caused by the avoided network upgrade (only $6 \%$ of increment with respect to the planning case without ESS).

\section{Conclusions}

The paper presents an innovative Multi-Objective approach for optimizing the implementation of energy storage systems on distribution networks. The main novelty proposed is the inclusion of the daily scheduling into the solution coding, together with siting and sizing of the storage devices, in order to simultaneously optimize all the main features that should be considered in a storage implementation project. This characteristic of the proposed methodology is essential to identify all the storage system configurations and their relative exploitation that represent a compromise among the contrasting goals and maximize the overall benefits brought by this technology. The developed tool gives the decision maker all the best design options once the goals and their relative metrics have been defined. Then application of the Decision Theory (e.g., Cost Benefit Analysis or Multi Criteria Analysis [27]) is necessary for identifying those options better suited to the planner needs. 


\section{Acknowledgment}

This research has been funded by the Italian Regulatory Authority for Electricity Gas and Water -

AEEGSI. The authors would like to thank Dr. L. Lo Schiavo and Dr. S. Larzeni for the technical

discussions and the deep review of reports that helped improve the final quality of results.

\section{References}

[1] European Commision (2017), "Energy storage - The role of electricity", SWD 61 Final.

[2] H. Saboori, R. Hemmati, S. M. Sadegh Ghiasi, S. Dehghan (2017), "Energy storage planning in electric power distribution networks - A state-of-the-art review", Renew. Sust. Energ. Rev., vol. 79, pp. 1108-1121.

[3] M. Zidar, P. S. Georgilakis, N. D. Hatziargyriou, T. Capuder, D. Skrlec (2016), "Review of energy storage allocation in power distribution networks: applications, methods and future research", IET Generation, Transmission and Distribution, vol. 10, no. 3, pp. 645-652.

[4] O. Babacan, W. Torre, J. Kleissi (2017), "Siting and sizing of distributed energy storage to mitigate voltage impact by solar PV in distribution systems", Solar Energy, vol. 146, pp. 199-208.

[5] G. Carpinelli, G. Celli, S. Mocci, F. Mottola, F. Pilo, D. Proto (2013), "Optimal Integration of Distributed Energy Storage Devices in Smart Grids”, IEEE Trans. on Smart Grid, vol. 4, no. 2, pp. 985-995.

[6] Y. Zheng, Z. Y. Dong, F. J. Luo, K. Meng, J. Qiu, K. P. Wong (2014), “Optimal allocation of Energy Storage System for risk mitigation of DISCOs with high renewable penetrations”, IEEE Trans. on Power Systems, vol. 29, no. 1, pp. 212-220.

[7] J.J. Jamian, M.W. Mustafa, H. Mokhlis, M.A. Baharudin (2014), "Simulation study on optimal placement and sizing of Battery Switching Station units using Artificial Bee Colony algorithm”, Int. Journal of Electric Power and Energy System, vol. 55, pp. 592-601.

[8] Y. Zhang, Z.Y. Dong, F. Luo, Y. Zheng, K. Meng, K.P. Wong (2016), “Optimal allocation of battery energy storage systems in distribution networks with high wind power penetration”, IET Renewable Power Generation, vol. 10, no. 8, pp. 1105-1113.

[9] Y. Atwa, EF. El-Saadany (2010), “Optimal allocation of ESS in distribution systems with a high penetration of wind energy”, IEEE Trans. on Power Systems, vol. 25, no. 4, pp. 1815-1822.

[10] M. Nick, R. Cherkaoui, M. Paolone (2014), "Optimal allocation of dispersed energy storage systems in active distribution networks for energy balance and grid support”, IEEE Trans. on Power Systems, vol. 29, no. 5, pp. 2300-2310.

[11] A. V. Pombo, J. Murta-Pina, V. F. Pires (2017), "Multiobjective formulation of the integration of storage systems within distribution networks for improving reliability", Electric Power System Research, vol. 148, pp. 87-96.

[12] M. Nick, R. Cherkaoui, M. Paolone (2015), "Optimal sizing and siting of distributed energy storage systems via alternating directional method of multipliers", Int. Journal of Electric Power Energy System, vol. 72, pp. 33-39.

[13] C. Coello Coello, D. A. Van Veldhuizen, G. B. Lamont (2002), "Evolutionary Algorithms for Solving MultiObjective Problems", Springer Science, New York.

[14] K. Deb, A. Pratap, S. Agarwal, T. Meyarivan (2002), “A Fast and Elitist Multiobjective Genetic Algorithm: NSGA-II”, IEEE Trans. on Evolutionary Computation, vol. 6, pp. 182-197.

[15] L. J. Eshelman, J. D. Schaffer (1993), "Real Coded Genetic Algorithms and Interval Schemata", in Foundation of Genetic Algorithms - vol. 2, L. Darrell Whitley (Ed.) (Morgan Kaufmann Publishers, San Mateo), pp. 187-202.

[16] G. Celli, S. Mocci, F. Pilo, G.G. Soma (2008), “A Multi-Objective Approach for the Optimal Distributed Generation Allocation with Environmental Constraints", in Proc. of PMAPS conference, Rincon (Porto Rico).

[17] D. E. Goldberg, B. L. Miller (1995), "Genetic Algorithms, Tournament Selection, and the Effects of Noise", Complex Systems, vol. 9, pp. 193-212.

[18] F. Herrera, M. Lozano, J.L. Verdegay (1998), "Tackling real-coded genetic algorithms: Operators and tools for behavioural analysis", in Artificial Intelligence Review, vol. 12, no. 4, pp. 265-319.

[19] F. Herrera, M. Lozano, A.M. Sanchez (2005), "Hybrid crossover operators for real-coded genetic algorithms: an experimental study", Soft Computing, vol. 9, pp. 280-298.

[20] K. Deb, D. Deb (2014), “Analyzing mutation schemes for Real-Parameter Genetic Algorithms", International Journal of Artificial Intelligence and Soft Computing, vol. 4, no. 1, pp. 1-28.

[21] C. Abbey, A. Baitch, B. Bak-Jensen, C. Carter-Brown, G. Celli, K. El Bakari, M. Fan, P. Georgilakis, T. Hearne, S. Jupe, N. Ochoa, G. Petretto, F. Pilo, F. Silvestro, J. Taylor (2014), "Planning and Optimization methods for Active Distribution Systems", Technical Report CIGRE' n. 591.

[22] G. Celli, E. Ghiani, F. Pilo, G.G. Soma, "New electricity distribution network planning approaches for integrating 
renewable", WIREs Energy and Environment, vol. 2, no. 2, pp. 140-157, 2013.

[23] G. Celli, F. Pilo, G.G. Soma, D. Dal Canto, E. Pasca, A. Quadrelli (2012), "Benefit assessment of energy storage for distribution network voltage regulation", in Proc. of CIRED Workshop, Lisbon (Portugal).

[24] G. Celli, F. Pilo, G. Pisano, G.G. Soma (2006), "Stochastic assessment of voltage dips for a PQ oriented distribution system development", in Proc. of PMAPS Conference, Stockholm (Sweden).

[25] A. Bracale, M. Coppo, F. Pilo, G. Pisano, R. Turri, et al. (2012), "Analysis of the Italian Distribution System Evolution through Reference Networks", in Proc. of IEEE ISGT Europe Conference, Berlin.

[26] A. Trivedi, D. Srinivasan, K. Sanyal, A. Ghosh (2017), "A survey of Multiobjective evolutionary algorithms based on decomposition", IEEE Trans. on Evolutionary Computation, vol. 21, no. 3, pp. 440-462.

[27] G. Celli, F. Pilo, G. Pisano, G.G. Soma (2017), "Cost/Benefit Analysis for energy storage exploitation in distribution systems", in Proc. of 24th CIRED conference, Glasgow (UK). 


\section{Figure captions}

Fig. 1 - Chromosome section for a storage device

Fig. 2 - Graphic representation of technical and operational constraints for the ESS scheduling

Fig. 3 - Graphical representation of the exploration and exploitation characteristics of a crossover operator for RCGA

Fig. 4 - Probability density function of creating a mutated offspring gene from a parent gene $c_{g}$

Fig. 5 - Flow chart of the network calculation procedure in presence of ESS

Fig. 6 - MV case study network

Fig. 7 - Occurrence probability of ESS siting among MV distribution network nodes (feeders marked with different colours)

Fig. 8 - Occurrence probability of ESS rating

Fig. 9 - Distributions of Pareto solutions with respect to each Objective Function (the zero points in the horizontal axes represent the performance of the distribution system without ESSs installed)

Fig. 10 - Example extracted from one of the best ESS allocations for network investment reduction (storage device of $2.1 \mathrm{MW}-8 \mathrm{~h}$, installed on node 62): a) stored energy profile, b) generable power duration for each hour of the day 


\section{List of Tables}

Table 1 - Parameters used for the nun-uniform mutation operator

\begin{tabular}{cccc}
\hline Gene & $\boldsymbol{a}_{\boldsymbol{g}}$ & $\boldsymbol{b}_{\boldsymbol{g}}$ & $\boldsymbol{\beta}$ \\
\hline$x_{\text {location }}$ & 1 & $N_{\text {nodes }}$ & 2 \\
$x_{P_{n}}$ & $P_{\min }$ & $P_{\max }$ & 5 \\
$x_{d_{n}}$ & $d_{\min }$ & $d_{\max }$ & 5 \\
$x_{S O C, h}$ & $\max \left\{0, x_{S O C, h-1}-P_{d}, x_{S o C, h+1}-P_{c}\right\}$ & $\min \left\{100, x_{S O C, h-1}+P_{c}, x_{S O C, h+1}+P_{d}\right\}$ & 5 \\
\hline
\end{tabular}

Table 2 -Objective Functions evaluation for the case study in the planning period without ESS

\begin{tabular}{cl}
\hline Objective Function & \multicolumn{1}{c}{ Performance } \\
\hline $\mathrm{OF}_{1}$ & $6428.9[\mathrm{k} €]$ \\
$\mathrm{OF}_{2}$ & $6862.9[\mathrm{MWh}]$ \\
$\mathrm{OF}_{3}$ & $18.7[$ p.u.] \\
$\mathrm{OF}_{4}$ & $0.923[$ hours/year·customer] \\
$\mathrm{OF}_{5}$ & $1960[$ voltage dips/year] \\
\hline
\end{tabular}




\section{List of Figures}

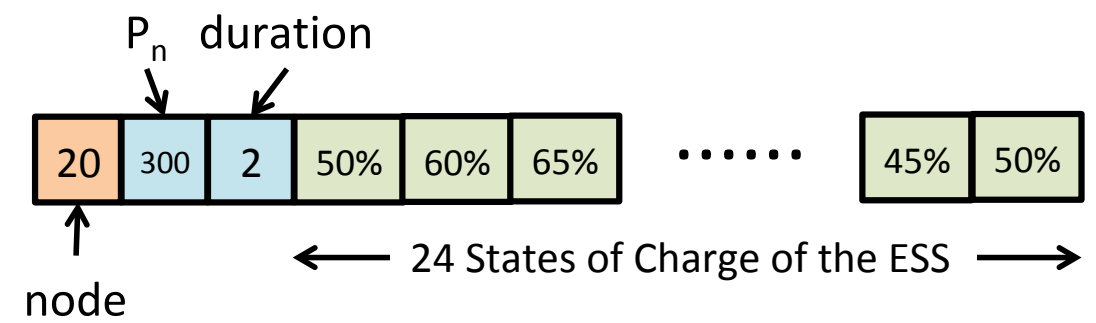

Fig. 1 - Chromosome section for a storage device

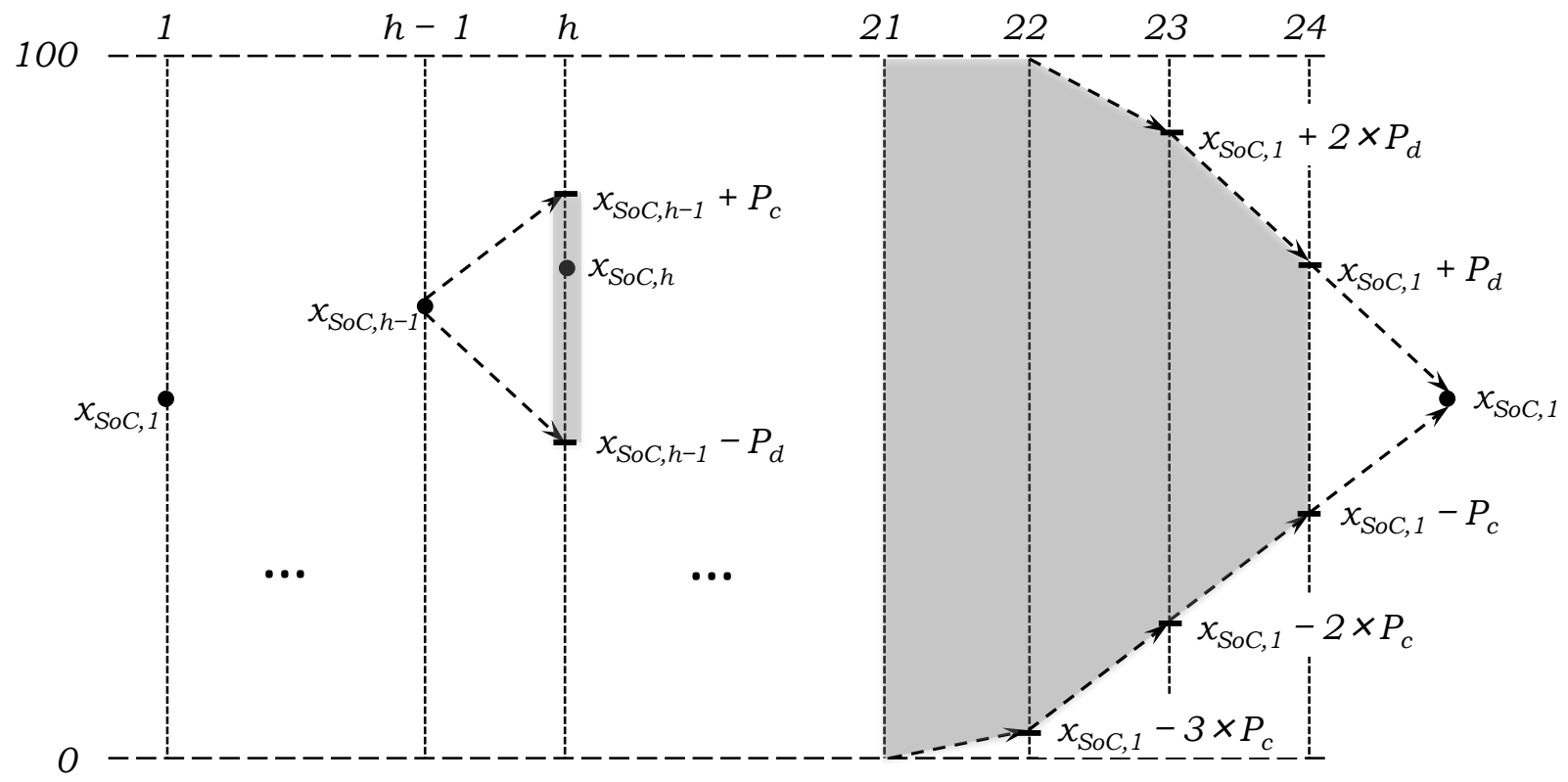

Fig. 2 - Graphic representation of technical and operational constraints for the ESS scheduling

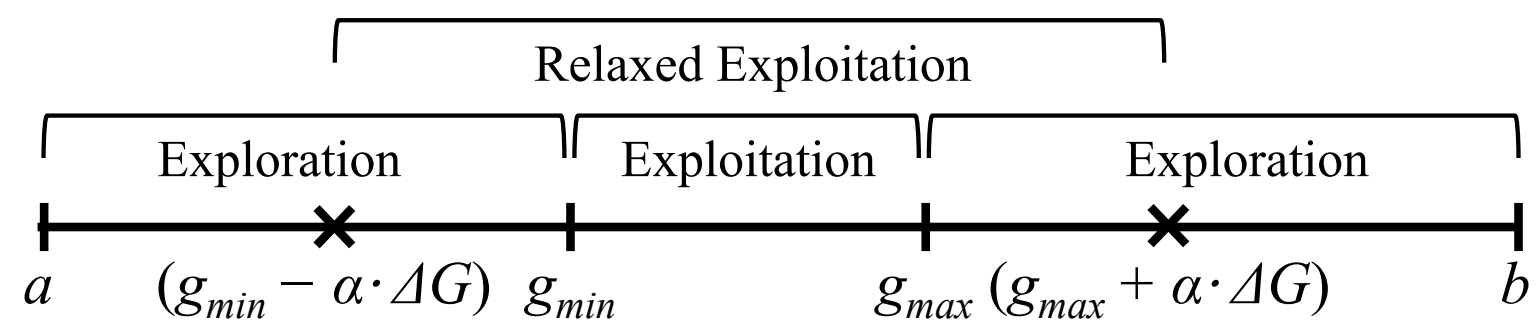

Fig. 3 - Graphical representation of the exploration and exploitation characteristics of a crossover operator for RCGA 
initial generations

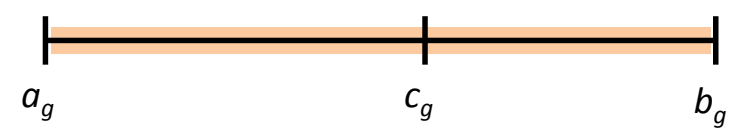

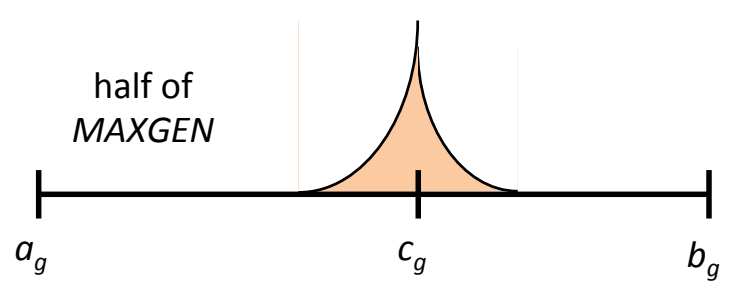

Fig. 4 - Probability density function of creating a mutated offspring gene from a parent gene $c_{g}$

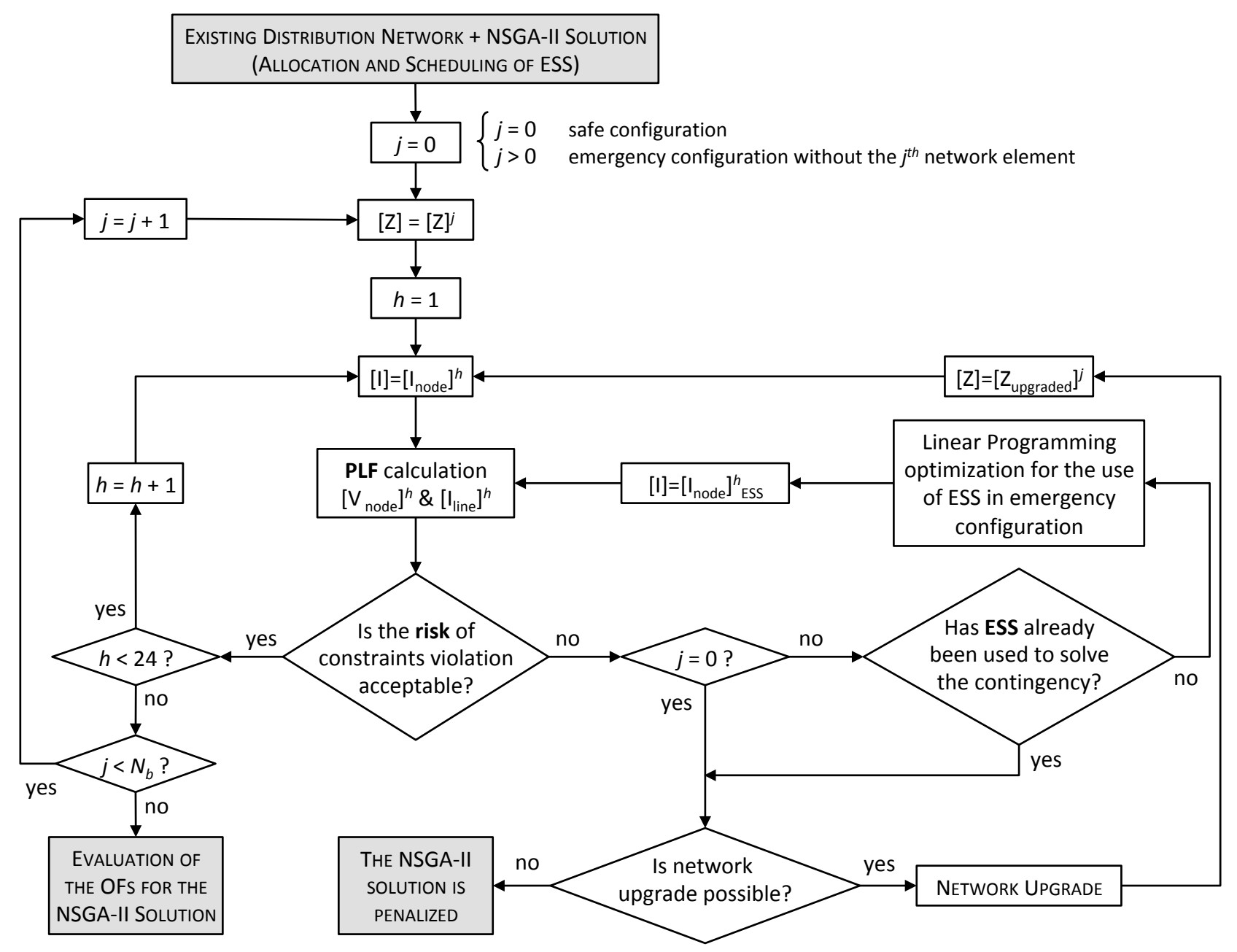

Fig. 5 - Flow chart of the network calculation procedure in presence of ESS 


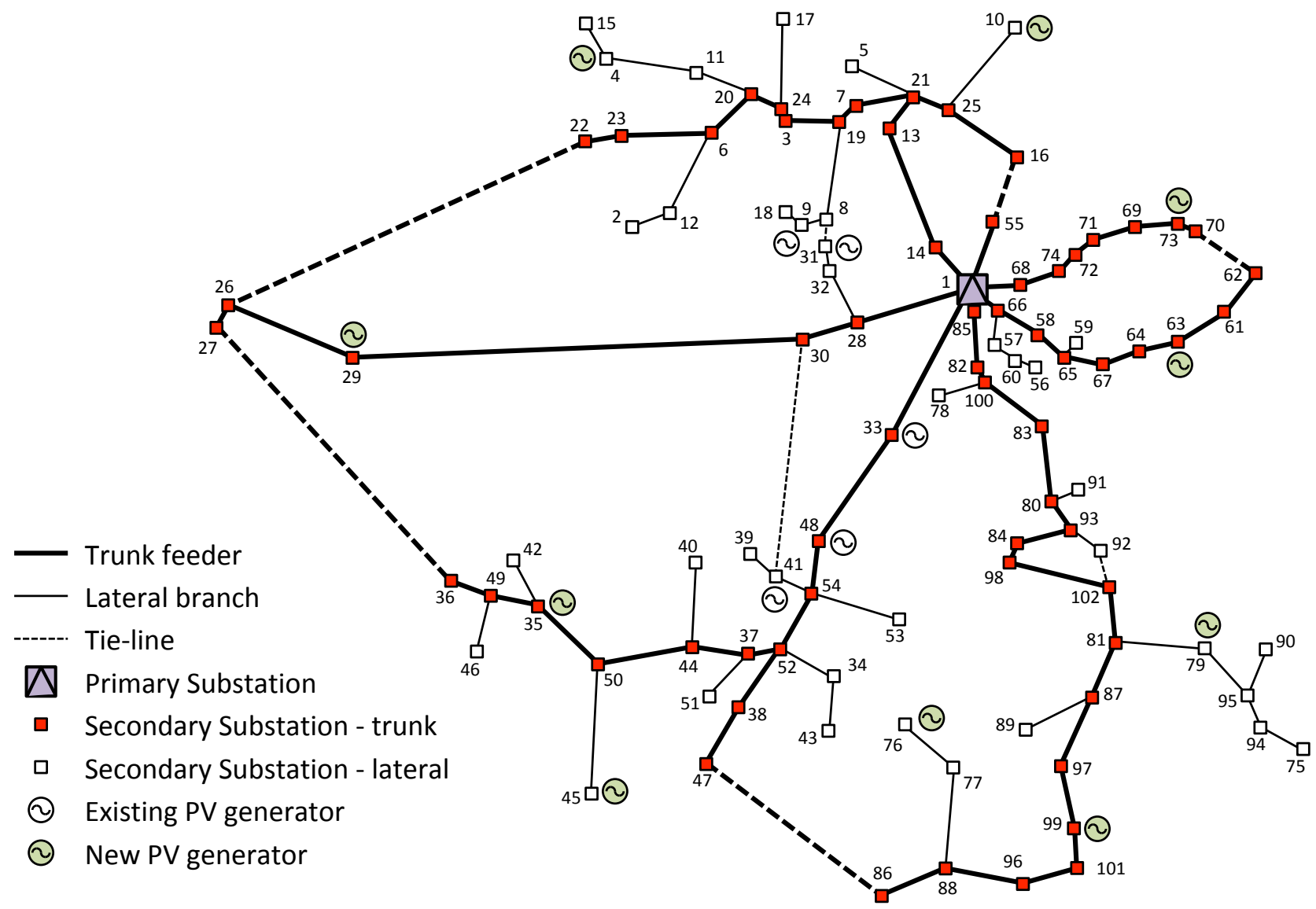

Fig. 6-MV case study network

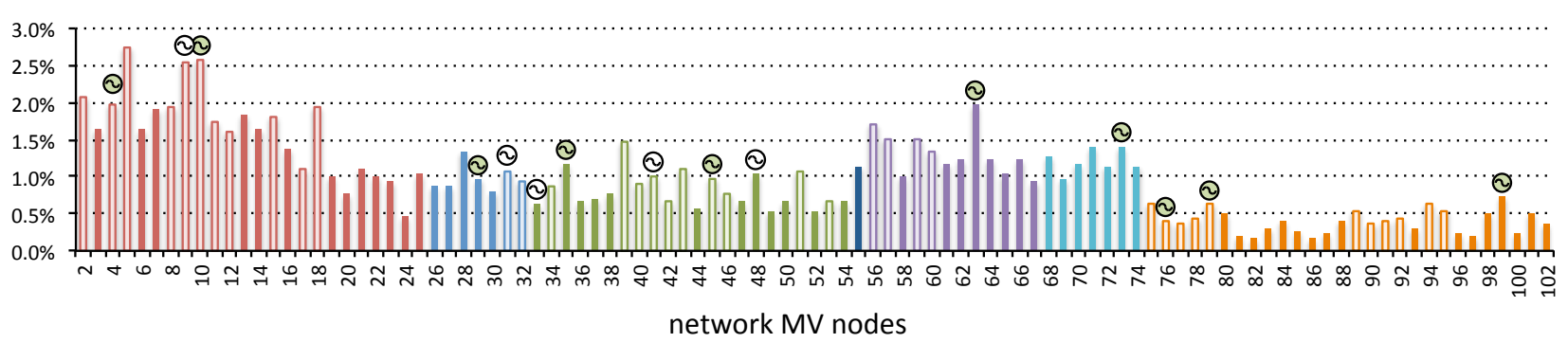

Fig. 7 - Occurrence probability of ESS siting among MV distribution network nodes (feeders marked with different colours) 


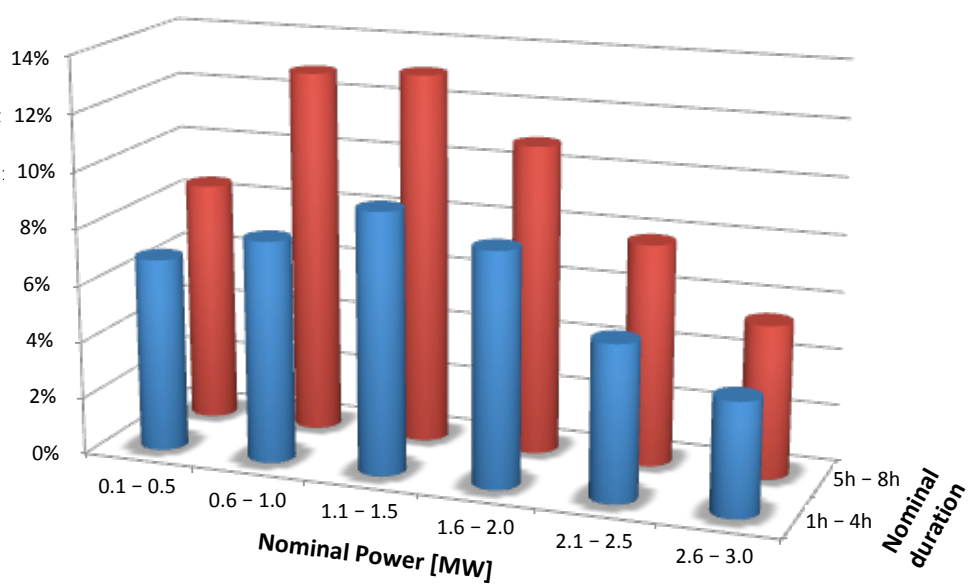

Fig. 8-Occurrence probability of ESS rating

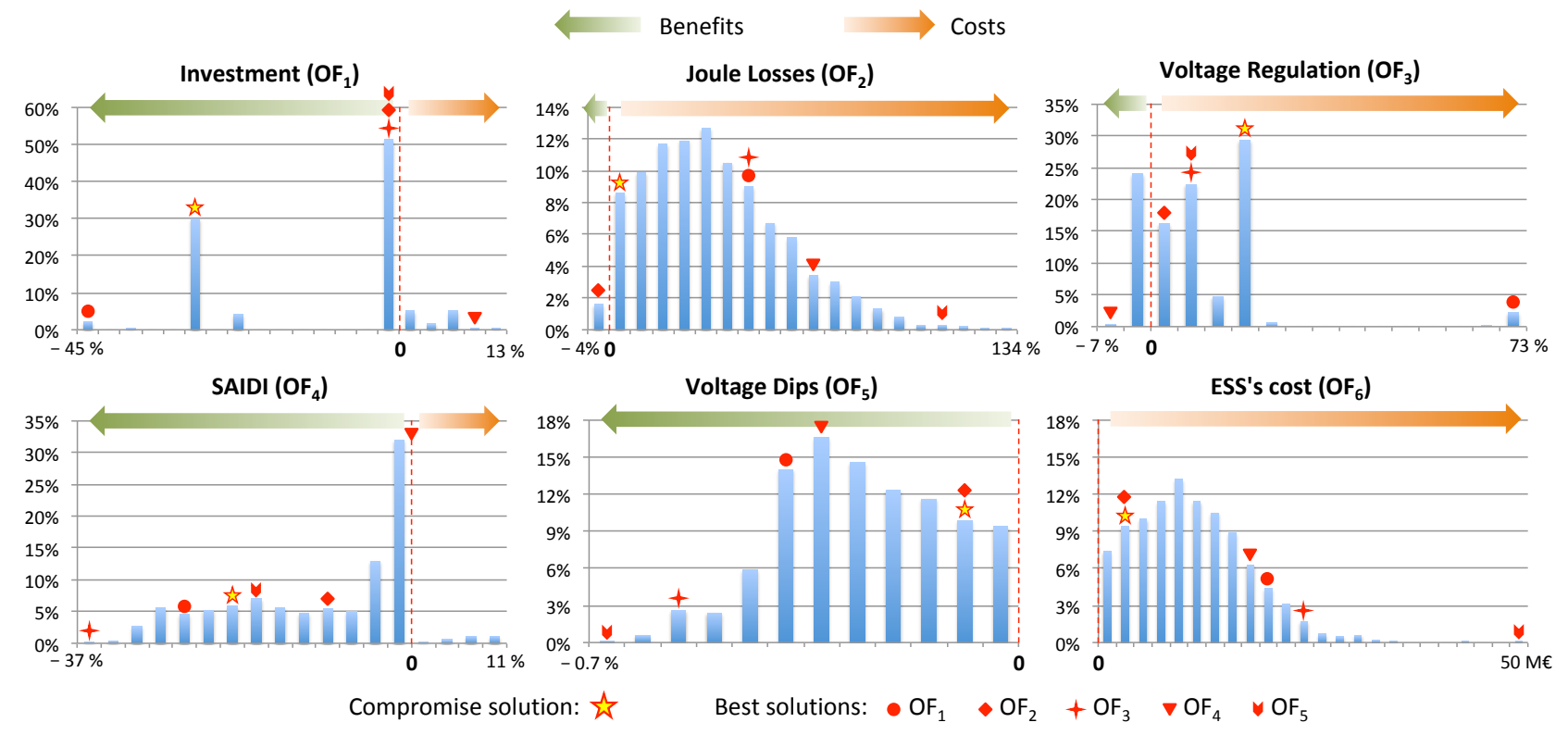

Fig. 9 - Distributions of Pareto solutions with respect to each Objective Function (the zero points in the horizontal axes represent the performance of the distribution system without ESSs installed)
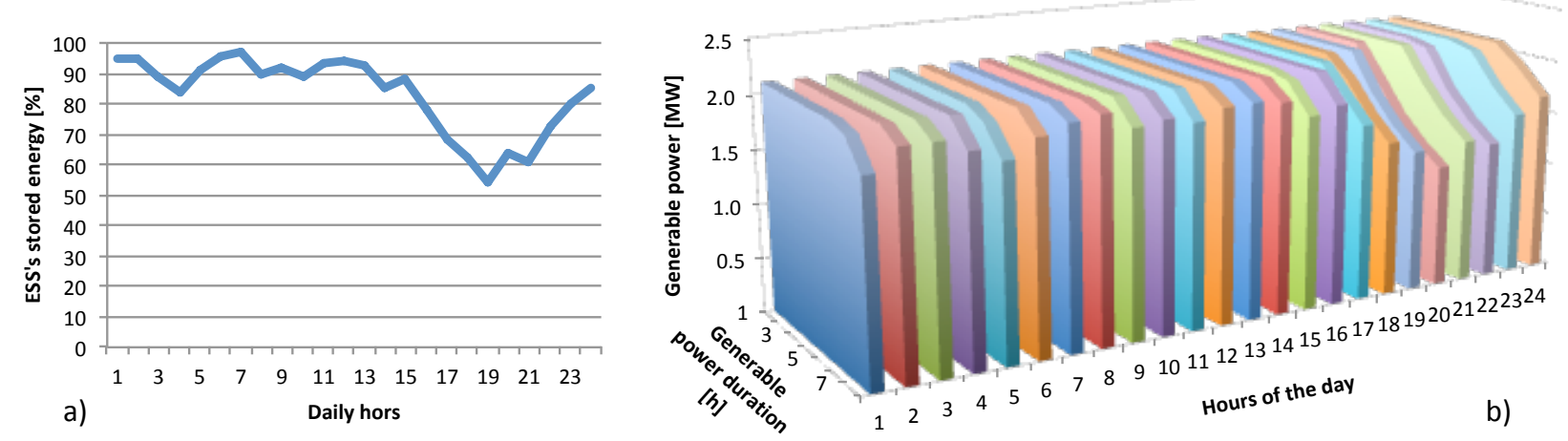

Fig. 11 - Example extracted from one of the best ESS allocations for network investment reduction (storage device of $2.1 \mathrm{MW}-8 \mathrm{~h}$, installed on node 62): a) stored energy profile, b) generable power duration for each hour of the day 